Pacific Journal of Mathematics

TOPOLOGIES WITH THE SAME CLASS OF 


\title{
TOPOLOGIES WITH THE SAME CLASS OF HOMEOMORPHISMS
}

\author{
YU-LEE LEE
}

Given a topological space $(X, \mathscr{Q})$, let $H(X, \mathscr{Q})$ be the class of all homeomorphisms of $(X, \mathscr{C})$ onto itself. This paper is devoted to study when and how a new topology $\mathscr{Y}$ can be constructed on $X$ such that $H(X, \mathscr{Q})=H(X, \mathscr{Y})$.

Everett and Ulam [1, 2] posed the problem in 1948. No results appeared until 1963, when J. V. Whittaker [3] proved the following.

Theorem. Suppose $X$ and $Y$ are compact, locally Euclidean manifolds (with or without boundary) and let $H(X)$ and $H(Y)$ be the groups of all homeomorphisms of $X$ and $Y$ onto themselves respectively. If $\alpha$ is a group isomorphism between $H(X)$ and $H(Y)$, then there exists a homeomorphism $\beta$ of $X$ onto $Y$ such that

$$
\alpha(h)=\beta h \beta^{-1} \quad \text { for all } h \in H(X) .
$$

From this theorem, we have immediately a partial answer to Ulam's problem.

Corollary. Suppose $(X, \mathscr{\mathscr { C }})$ and $(X, \mathscr{Y})$ are compact, locally Euclidean manifolds, with or without boundary, with the class of homeomorphisms $H(X, \mathscr{C})$ and $H(X, \mathscr{V})$ respectively. If $H(X, \mathscr{U})=$ $H\left(X, \mathscr{V}^{\prime}\right)$, then $(X, \mathscr{U})$ is homeomorphic to $(X, \mathscr{Y})$.

But there do exist many topologies $\mathscr{Y}^{n}$ such that $H(X, \mathscr{U})=$ $H\left(X, \mathscr{V}^{\prime}\right)$ and $(X, \mathscr{U})$ is not homeomorphic with $(X, \mathscr{V})$. The purpose of this paper is to construct such topologies. There exist many nonhomeomorphic topologies for the real line which possess the same class of homeomorphisms as the real line with its usual topology.

2. Let $(X, \mathscr{U})$ be a topological space and $A$ a subset of $X$. We denote the class of all homeomorphisms of $(X, \mathscr{U})$ onto itself by $H(X, \mathscr{\ell})$ and set

$$
H(A)=\{f(x): f \in H(X, \mathscr{U}), x \in A\} .
$$

We shall construct many new topologies $\mathscr{Y}_{\alpha}^{\alpha}(\alpha \in D)$ for $X$ such that $H(X, \mathscr{U})=H\left(X, \mathscr{V}_{\alpha}\right)$ for each $\alpha \in D$. By $X-A, \mathrm{Cl}(A)$, Int $(A)$, and $\operatorname{Bndy}(A)$, unless otherwise stated, we always mean the complement, closure, interior, and the boundary of $A$ with respect to the original topology $\mathscr{C}$. 
Lemma 1. Let $(X, \mathscr{Q})$ be a topological space and let $P(V)$ be a topological property possessed by certain subsets $V$ of $X$. If $\mathscr{V}^{\circ}=$ $\{V: P(V)\}$ is a topology for $X$, then $H(X, \mathscr{Q}) \subset H\left(X, \mathscr{Y}^{-}\right)$.

Proof. Trivial.

Lemma 2. Let $\mathscr{Q}$ and $\mathscr{Y}$ be two topologies for $X$ such that $U \in \mathscr{Q}$ if and only if $U \cup V \in \mathscr{Y}$ for all nonempty $V$ in $\mathscr{Y}$. Then $H(X, \mathscr{\ell}) \supset H(X, \mathscr{V})$.

Proof. Suppose $f \in H(X, \mathscr{V}), U \in \mathscr{C}$ and $V \neq \varnothing$ in $\mathscr{V}$. Then

$$
f^{-1}(V \cup f(U))=f^{-1}(V) \cup U \in \mathscr{q}
$$

Hence

$$
V \cup f(U) \in \mathscr{Y} \text { and } f(U) \in \mathscr{U} \text {. }
$$

Therefore $f^{-1}$ is a continuous mapping of $(X, \mathscr{U})$ onto itself, and a similar argument proves that $f$ is a continuous mapping of $(X, \mathscr{C})$ onto itself. Therefore $f \in H(X, \mathscr{Q})$.

THeOREM 3. Let $(X, \mathscr{Q})$ be a locally compact space. Let $P(V)$ mean that $V \in \mathscr{Q}$ and $X-V$ is closed and compact. Then

$$
\mathscr{V}^{\prime}=\{U: U=\varnothing \text { or } P(U)\}
$$

is a topology for $X$ and $H(X, \mathscr{U})=H(X, \mathscr{V})$.

Proof. It is clear that $\mathscr{Y}$ is a topology for $X$ and, by Lemma 1 , $H(X, \mathscr{Q}) \subset H(X, \mathscr{V})$.

If $(X, \mathscr{C})$ is compact, then $\mathscr{U}=\mathscr{V}$. Therefore we may assume that $(X, \mathscr{U})$ is not compact.

If $U \in \mathscr{U}, V \neq \varnothing$ and $V \in \mathscr{Y}$; then $X-V$ is closed and compact in $(X, \mathscr{U})$, and hence $X-(U \cup V)$ is closed and compact in $(X, \mathscr{U})$. Therefore $U \cup V \in \mathscr{Y}$.

Conversely, suppose $U \notin \mathscr{U}$. Then there exists $x$ in $U-\operatorname{Int}(U)$ and a neighborhood $W$ of $x$ such that $\mathrm{Cl}(W)$ is compact in $(X, \mathscr{U})$ and $\mathrm{Cl}(W) \neq X$. Moreover $x \in \mathrm{Cl}(\mathrm{Cl}(W)-U)$. Hence $X-\mathrm{Cl}(W)$ is a nonempty element of $\mathscr{Y}$, and $(X-\mathrm{Cl}(W)) \cup U \notin \mathscr{U}$. This implies that $X-\mathrm{Cl}(W) \cup U \notin \mathscr{V}$ since $\mathscr{V} \subset \mathscr{U}$.

By Lemma 2, we have $H(X, \mathscr{U}) \subset H(X, \mathscr{Y})$.

The following corollary is a generalization of Theorem 3 , but the pattern of proof is essentially the same.

Corollary 4. Let $(X, \mathscr{U})$ be a locally compact or a regular 
space and let $A$ be a closed locally compact subset of $X$ such that $f(A)=A$ for all $f$ in $H(X, \mathscr{Q})$. Let $P(V)$ mean that $V \in \mathscr{Q}$ and $A-V=B \cup C$ where $B$ is a closed and compact set and $C$ is a closed and nowhere dense set and $\operatorname{Card}(C) \leqq \alpha$ for some fined infinite cardinal number $\alpha$. Then

$$
\mathscr{V}=\{U: U=\varnothing \text { or } P(U)\}
$$

is a topology for $x$ and $H(X, \mathscr{Q})=H\left(X, \mathscr{Y}^{\prime}\right)$.

Proof. It is easy to check that $\mathscr{V}^{-}$is a topology for $X$ and $P(U)$ is a topological property and by Lemma $1, H(X, \mathscr{U}) \subset H(X, \mathscr{V})$.

If $(X, \mathscr{U})$ is compact, then $A$ and $A-V$ are closed compact sets for any open set $V$. Hence $A-V=(A-V) \cup \varnothing$ and $\mathscr{Q}=\mathscr{Y}$. Therefore we may assume that $(X, \mathscr{U})$ is locally compact but not compact. If $U \in \mathscr{W}$, then it is clear that $U \cup V \in \mathscr{Y}$ for any nonempty $V$ in $\mathscr{Y}$. If $U \notin \mathscr{Q}$, then there exists $x$ in $U-\operatorname{Int}(U)$. Let $W$ be a closed compact neighborhood of $x$ we have $W \neq X$ and $x \in \mathrm{Cl}(W-U)$. Since $A-(X-W)=(A \cap W)$ is closed compact, $X-W$ is a nonempty element of $\mathscr{Y}$ and $(X-W) \cup U \notin \mathscr{U}$. This implies that $(X-W) \cup U \notin \mathscr{Y}$ since $\mathscr{Y} \subset \mathscr{Q}$. By Lemma 2 we have that $H(X, \mathscr{U})=H(X, \mathscr{Y})$.

If $(X, \mathscr{C})$ is regular, then $U \in \mathscr{C}$ implies $U \cup V \in \mathscr{Y}$ for all nonempty $V$ in $\mathscr{V}$ is still true. If $U \notin \mathscr{L}$, then there exists $x$ in $U$ - Int $(U)$. If $x \in A$, then since $A$ is a locally compact subset of $(X, \mathscr{C})$, the argument is the same as above. If $x \notin A$, then there exists a closed neighborhood $W_{x}$ of $x$ such that $W_{x} \cap A=\varnothing$. Hence $A-\left(X-W_{x}\right)=A \cap W_{x}=\varnothing$ and $X-W_{x}$ is a nonempty element in $\mathscr{V}$. But $\left(X-W_{x}\right) \cup U$ is not in $\mathscr{C}$, hence it is also not in $\mathscr{V}$.

THEOREM 5. Let $A$ be a closed subset of a first countable Hausdorff space $(X, \mathscr{Q})$ such that $f(A)=A$ for each $f$ in $H(X, \mathscr{Q})$ and $A$ contains no isolated point relative to the relative topology and suppose the set $I$ of all isolated points of $\left(X, \mathscr{Q}_{6}\right)$ is closed. If

$$
\mathscr{V}=\{U \in \mathscr{Q}: U=\varnothing \quad \text { or } \quad \mathrm{Cl}(U \cap A)=A\},
$$

then $(X, \mathscr{Y})$ is a topological space and $H(X, \mathscr{C})=H(X, \mathscr{Y})$.

If $A=X$, then the condition that $A$ contains no isolated point can be dropped.

Proof. It is clear that $(X, \mathscr{Y})$ is a topological space and, by Lemma $1, H(X, \mathscr{U}) \subset H(X, \mathscr{Y})$. Also if $U \in \mathscr{Q}$ and $V \neq \varnothing$ in $\mathscr{Y}$, then $U \cup V \in \mathscr{Y}$. 
Suppose $U \notin \mathscr{C}$. Then there exists $p_{0}$ in $U$ and a sequence $\left\{p_{i}\right\}$ in $X-U$ such that $\left\{p_{i}\right\}$ converges to $p_{0}$ and $p_{i} \notin I$ for each $i$. Therefore

$$
\left(X-\left\{p_{i}: i=0,1,2, \cdots\right\}\right) \in \mathscr{V}
$$

but

$$
U \cup\left(X-\left\{p_{i}: i=0,1,2, \cdots\right\}\right) \notin \mathscr{Y} .
$$

Hence $U \in \mathscr{Q}$ if and only if $U \cup V \in \mathscr{Q}$ for all nonvoid $V$ in $\mathscr{Y}$. $B y$ Lemma 2, we have $H(X, \mathscr{U}) \supset H(X, \mathscr{V})$.

By similar arguments, we can prove the following theorem since in each case the only property required is that $X-\left\{p_{i}: i=0,1,2, \cdots\right\} \in \mathscr{Y}$.

Theorem 6. Let $(X, \mathscr{U}), A$ and $I$ be as in Theorem 5. Let $P_{1}(V)$ mean that $V \in \mathscr{Q}, \mathrm{Cl}(V \cap A)=A$ and $A-V$ is compact, $P_{2}(V)$ mean that $V \in \mathscr{L}, \mathrm{Cl}(V \cap A)=A$ and $A-V$ is countably compact, $P_{3}(V)$ mean that $V \in \mathscr{U}, \mathrm{Cl}(V \cap A)=A$ and $\operatorname{Card}(A-V) \leqq \alpha$ where $\alpha$ is any fixed cardinal number greater than or equal to $\aleph_{0}$, and $P_{4}(V)$ mean that $V \in \mathscr{Q}, \mathrm{Cl}(V \cap A)=A$, Card $(A-V) \leqq \alpha$ and $A-V$ is compact. Then $\mathscr{V}_{i}=\left\{U: U=\varnothing\right.$ or $\left.P_{i}(U)\right\}(i=1,2,3,4)$ are topologies and $H(X, \mathscr{U})=H\left(X, \mathscr{V}_{i}\right)(i=i, 2,3,4)$.

The condition that the set $I$ of all isolated points in $(X, \mathscr{U})$ be closed is necessary in the above theorems. For let

$$
X=[-1,0] \cup\left\{\frac{1}{n}: n=1,2, \cdots\right\}
$$

with the relative topology inherited from the real line, and let $A=$ $[-1,0]$. Let $f(x)=-x-1$ when $x \in[-1,0]$ and $f(1 / n)=1 / n$ for all n. Then $f \in H\left(X, \mathscr{Y}_{i}\right)$ and $f \in H(X, \mathscr{y})$ but $f \notin H(X, \mathscr{Q})$. But we can set another condition in order to permit that $I$ has exactly one limit point.

TheOREM 7. Let $A$ be a closed subset of a first countable Hausdorff space $(X, \mathscr{U})$ such that $f(A)=A$ for each $f$ in $H(X, \mathscr{U})$ and $A$ contains no isolated points relative to the relative topology and suppose the set $I$ of all isolated points of $(X, \mathscr{Q})$ has exactly one limit point $e$ and $I$ is compact. Suppose in addition that $f(e)=e$ for any $f$ in $H(X-I, \mathscr{Q} \mid X-I)$. If

$$
\mathscr{V}=\{U \in \mathscr{Q}: U=\varnothing \text { or } \mathrm{Cl}(U \cap A\rangle=A\},
$$

then $(X, \mathscr{V})$ is a topological space and $H(X, \mathscr{U})=H(X, \mathscr{V})$.

If $A=X$, then the condition that $A$ contain no isolated points 
can be dropped.

Proof. It is clear that $\mathscr{Y}^{-}$is a topology for $X$ and by Lemma 1 , $H(X, \mathscr{Q}) \subset H(X, \mathscr{V})$.

Let $f \in H\left(X, \mathscr{V}^{\circ}\right)$. Since $\{p\} \cup V \in \mathscr{Y}$ for all nonvoid $V$ in $\mathscr{V}^{-}$ if and only if $p \in I$, and in case $A=X, p \in V$ for all nonvoid $V$ in $\mathscr{Y}^{-}$if and only if $p \in I$, it follows that $f(I)=I$ and hence $f \mid(X-I) \in H(X-I, \mathscr{V} \mid X-I)$. If $e$ is not an isolated point of $X-I$, by Theorem $5, H(X-I, \mathscr{Y} \mid X-I)=H(X-I, \mathscr{Q} \mid X-I)$ and hence $f(e)=e$. If $f \notin H(X, \mathscr{Q})$, then either there exists a sequence $\left\{p_{i}\right\}$ and $p_{0}$ in $X$ such that $\left\{p_{i}\right\}$ converges to $p_{0}$ but $\left\{f\left(p_{i}\right)\right\}$ does not converge to $f\left(p_{0}\right)$, or there exists a sequence $\left\{p_{i}\right\}$ and $p_{0}$ in $X$ such that $\left\{p_{i}\right\}$ does not converge to $p_{0}$ but $\left\{f\left(p_{i}\right)\right\}$ converges to $f\left(p_{0}\right)$. In the first case, if $p_{0} \neq e$, we may choose $p_{i} \notin I$ for each $i$. Then $\left\{p_{i}\right\}$ converges to $p_{0}$ in $(X-I, \mathscr{C} \mid X-I)$ and $\left\{f\left(p_{i}\right)\right\}$ does not converge to $f\left(p_{0}\right)$ in $(X-I, \mathscr{Q} 6 \mid X-I)$ and hence

$$
f \mid X-I \notin H(X-I, \mathscr{Q} \mid X-I)=H(X-I, \mathscr{V} \mid X-I) .
$$

This is a contradiction. If $p_{0}=e$, then since $I$ is compact, every $\mathscr{U}$ neighborhood of $e$ contains all but a finite number of the points of $I$. If all subsequences of $\left\{p_{i}\right\}$ are in $I$, then $\left\{f\left(p_{i}\right)\right\}$ converges to $f(e)=e$. If there is a subsequence $\left\{p_{n_{i}}\right\} \subset X-I$ such that $\left\{p_{n_{i}}\right\}$ converges to $e$ but $\left\{f\left(p_{n_{i}}\right)\right\}$ does not converge to $e$, then $f \notin H(X-I, \mathscr{Q} \mid X-I)$. This is also a contradiction. Therefore $f$ is a continuous function of $(X, \mathscr{Q})$ onto itself. By a similar argument $f^{-1}$ is continuous. Therefore $H(X, \mathscr{Q})=H(X, \mathscr{V})$. If $e$ is an isolated point of $X-I$, then $e \notin A$ and $e$ is the only point in $X$ such that every $\mathscr{Y}$-neighborhood of $e$ contains all but finite points of $I$, but in case $X=A$, $e$ is the only point in $X-I$ such that $V \in \mathscr{Y}$ if and only if $V \cup\{e\} \in \mathscr{Y}$ for any nonempty $V$ in $\mathscr{Y}$. Hence $f(e)=e$. A similar argument applies to $H(X-\bar{I}, \mathscr{Y} \mid X-\bar{I})$, and we also have $H(X, \mathscr{C})=H(X, \mathscr{Y})$.

We can see that the theorem analogous to Theorem 6 also holds.

Theorem 8. Let $(X, \mathscr{Q}), A, I$, and $e$ be as in Theorem 7. Let $P_{1}(V)$ mean that $V \in \mathscr{U}, \mathrm{Cl}(V \cap A)=A$ and $A-V$ is compact, $P_{2}(V)$ mean that $V \in \mathscr{U}, \mathrm{Cl}(V \cap A)=A$ and $A-V$ is countably compact, $P_{3}(V)$ mean that $V \in \mathscr{L}, \mathrm{Cl}(V \cap A)=A$ and $\operatorname{Card}(A-V) \leqq \alpha$ where $\alpha$ is any fixed cardinal number greater than or equal to $\boldsymbol{\aleph}_{0}$. Then $\mathscr{V}_{i}=\left(U: U=\varnothing\right.$ or $P_{i}(U)(i=i, 2,3)$ are topologies and $H(X, \mathscr{C})=$ $H\left(X, \mathscr{Y}_{i}\right)(i=1,2,3)$.

If $A=X$, then the condition that $A$ contain no isolated points can be omitted. 
In Theorem 7 and Theorem 8, the condition $f(e)=e$ for any $f$ in $H(X-I, \mathscr{C} \mid X-I)$ is necessary as shown in the preceding example. If $X=A$, then the condition that $\bar{I}$ be compact is also necessary since otherwise there exists a neighborhood $U$ of $e$ such that Card $(I-U) \geqq$ $\aleph_{0}$ and any one-to-one mapping $f$, such that

$$
f \mid X-I \in H(X-I, \mathscr{Q} \mid X-I)
$$

and such that $f$ maps a sequence of points in $I-U$ to a sequence of points converging to $e$, is in $H(X, \mathscr{V})-H(X, \mathscr{C})$. And if $I$ has more than one limit point, let $e_{1}$ and $e_{2}$ be any two of them. Then any one-to-one mapping $f$ such that $f \mid X-I \in H(X-I, \mathscr{Q} \mid X-I)$ and such that $f$ interchanges a sequence of points $\left\{p_{i}\right\}$ converging to $e_{1}$ and a sequence of points $\left\{q_{i}\right\}$ converging to $e_{2}$ is in $H(X, \mathscr{Y})-H(X, \mathscr{C})$. But if $A \neq X$, the conditions that $\bar{I}$ be compact and that $I$ has exactly one limit point can be dropped with a little modification of proof. However we have the following theorem.

THEOREM 9. Let $(X, \mathscr{C})$ be a compact Hausdorff space and let $p_{0} \in X$ such that $f\left(p_{0}\right)=p_{0}$ for any $f$ in $H(X, \mathscr{Q})$. Let $P(U)$ mean that $U \in \mathscr{U}, p_{0} \notin U$ and $\left(X-p_{0}\right)-U$ is compact. If

$$
\mathscr{V}=\{U: U=X \text { or } U=Q \text { or } P(U)\},
$$

then $(X, \mathscr{Y})$ is a topological space and $H(X, \mathscr{U})=H(X, \mathscr{Y})$.

Proof. It is clear that $(X, \mathscr{C})$ is a topological space and $H(X, \mathscr{C}) \subset H(X, \mathscr{Y})$ by Lemma 1 . If $p_{0}$ is an isolated point, it is also clear that $H(X, \mathscr{C})=H(X, \mathscr{Y})$. Hence we may assume that $p_{0}$ is not an isolated point.

Let $A \subset X-p_{0}$, then $A$ is closed and compact in $(X, \mathscr{C})$ if and only if $A$ is closed and compact in $\left(X-p_{0}, \mathscr{C} \mid X-p_{0}\right)$.

If $f \in H(X, \mathscr{Y})$, then $f\left(p_{0}\right)=p_{0}$, since $p_{0}$ is the only point in $(X, \mathscr{Y})$ such that $p_{0}$ has exactly one neighborhood, namely $X$. Hence $f \mid X-p_{0} \in H\left(X-p_{0}, \mathscr{V} \mid X-p_{0}\right)$. By Theorem 3 and the above discussion, $f \mid X-p_{0} \in H\left(X-p_{0}, \mathscr{C} \mid X-p_{0}\right)$. If $U \in \mathscr{Q}$ and $p_{0} \in U$, then $U \in \mathscr{U} \mid X-p_{0}$ and hence $f(U) \in \mathscr{U} \mid X-p_{0}$ and $f(U) \in \mathscr{W}$. If $p_{0} \in U \in \mathscr{U}$, then $X-U$ is closed and compact in $\left(X-p_{0}, \mathscr{Q} \mid X-p_{0}\right)$ and $f(X-U)=$ $X-f(U)$ is closed and compact in $\left(X-p_{0}, \mathscr{Q} \mid X-p_{0}\right)$. It follows that $f(U) \in \mathscr{C}$ and similarly $f^{-1}(U) \in \mathscr{U}$. Hence we have $H(X, \mathscr{C})=H(X, \mathscr{V})$.

THEOREM 10. Let $(X, \mathscr{Q})$ be a compact Hausdorff space without isolated points and let $p_{0} \in X$ such that $f\left(p_{0}\right)=p_{0}$ for any $f$ in $H(X, \mathscr{Q})$. Let $P(V)$ mean that $V \in \mathscr{Q}$ and $p_{0} \in V$ or $V \in \mathscr{Y}$ and $\left(X-p_{0}\right)-V$ compact. If 


$$
\mathscr{V}^{n}=\{U: U=\varnothing \text { or } P(U)\},
$$

then $(X, \mathscr{Y})$ is a topological space and $H(X, \mathscr{Q})=H(X, \mathscr{V})$.

Proof. The argument is similar to that in Theorem 9. The only thing we have to show is that $f\left(p_{0}\right)=p_{0}$ for any $f$ in $H(X, \mathscr{Y})$, and this follows from the fact that $p_{0}$ is the only point in $X$ such that $\left\{p_{0}\right\} \cup V \in \mathscr{V}^{-}$for any nonvoid $V$ in $\mathscr{Y}$.

The author wishes to acknowledge his debt and gratitude to Professor Lewis E. Ward, Jr., for his constant encouragement and helpful suggestions during the preparation of this paper. I am graterul to the referee for many suggestions which improve this paper a great deal.

\section{BIBLIOGRAPHY}

1. C. J. Everett and S. M. Ulam, On the problem of determination of mathematical structures by their endomorphisms, Bull. Amer. Math. Soc. 54 (1948), Abstract 285t. 2. S. M. Ulam, A Collection of Mathematical Problems, Interscience Publishers, New York, 1960.

3. J. V. Whittaker, On isomorphic groups and homeomorphic spaces, Ann. of Math. 78 (1963), 74-91.

4. Yu-Lee Lee, Dissertation, University of Oregon, Eugene, 1964.

5. Yu-Lee Lee, Characterizing the topology by the class of homeomorphisms, (to appear)

Received June 7, 1965. This paper is a portion of the author's dissertation at the University of Oregon, 1964. This research was supported by the National Science Foundation, under grant number GP-1457.

UNIVERSITY OF FLORIDA AND

UNIVERSITY OF CONNECTICUT 



\section{PACIFIC JOURNAL OF MATHEMATICS}

H. SAMELSON

Stanford University

Stanford, California

J. P. JaNs

University of Washington

Seattle, Washington 98105

\section{EDITORS}

J. DugundJI

University of Southern California Los Angeles, California 90007

RICHARD ARENS

University of California

Los Angeles, California 90024

\section{ASSOCIATE EDITORS}

E. F. BECKENBACH

B. H. NEUMANN

F. WOLF

K. YosidA

\section{SUPPORTING INSTITUTIONS}

UNIVERSITY OF BRITISH COLUMBIA

CALIFORNIA INSTITUTE OF TECHNOLOGY

UNIVERSITY OF CALIFORNIA

MONTANA STATE UNIVERSITY

UNIVERSITY OF NEVADA

NEW MEXICO STATE UNIVERSITY

OREGON STATE UNIVERSITY

UNIVERSITY OF OREGON

OSAKA UNIVERSITY

UNIVERSITY OF SOUTHERN CALIFORNIA
STANFORD UNIVERSITY

UNIVERSITY OF TOKYO

UNIVERSITY OF UTAH

WASHINGTON STATE UNIVERSITY

UNIVERSITY OF WASHINGTON

AMERICAN MATHEMATICAL SOCIETY CHEVRON RESEARCH CORPORATION TRW SYSTEMS

NAVAL ORDNANCE TEST STATION 


\section{Pacific Journal of Mathematics}

\section{Vol. 20, No. $1 \quad$ September, 1967}

Leonard Daniel Baumert, Extreme copositive quadratic forms. II ........ 1

Edward Lee Bethel, A note on continuous collections of disjoint

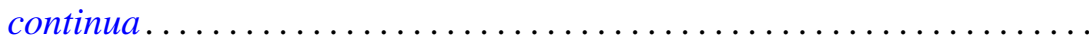

Delmar L. Boyer and Adolf G. Mader, A representation theorem for abelian groups with no elements of infinite p-height ...................

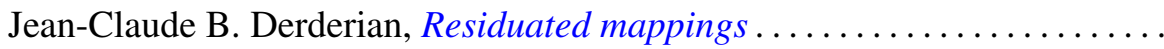

Burton I. Fein, Representations of direct products of finite groups ......... 45

John Brady Garnett, A topological characterization of Gleason parts.......

Herbert Meyer Kamowitz, On operators whose spectrum lies on a circle or

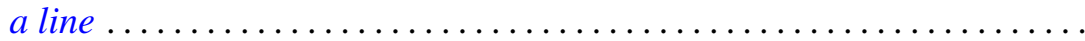

Ignacy I. Kotlarski, On characterizing the gamma and the normal distribution ........................................

Yu-Lee Lee, Topologies with the same class of homeomorphisms ..........

Moshe Mangad, Asymptotic expansions of Fourier transforms and discrete polyharmonic Green's functions ...........................

Jürg Thomas Marti, On integro-differential equations in Banach spaces ....

Walter Philipp, Some metrical theorems in number theory.............. 109

Maxwell Alexander Rosenlicht, Another proof of a theorem on rational

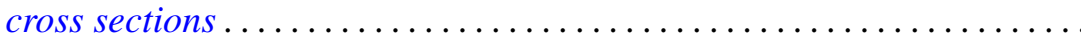

Kenneth Allen Ross and Karl Robert Stromberg, Jessen's theorem on Riemann sums for locally compact groups

Stephen Simons, A theorem on lattice ordered groups, results of Ptak, Namioka and Banach, and a front-ended proof of Lebesgue's theorem...

Morton Lincoln Slater, On the equation $\varphi(x)=\int_{x} x+1 K(\xi) f[\varphi(\xi)] d \xi \ldots$ 155 Arthur William John Stoddart, Existence of optimal controls .. 167 Burnett Roland Toskey, A system of canonical forms for rings on a direct sum of two infinite cyclic groups ....................

Jerry Eugene Vaughan, A modification of Morita's characterization of dimension 